\title{
Ten Tips for Blended Practicals
}

\author{
Garrard, A ${ }^{1}$., Di Benedetti, M., Bangert, K., Funnell, A. C., and Beck, S \\ All authors are from: The Department of Multidisciplinary Engineering Education, The \\ University of Sheffield, Faculty of Engineering, The Diamond, Sheffield, S3 7RD, UK
}

Keywords: practical engineering education; remote practicals; blended learning

\begin{abstract}
At the start of 2020 the rapid onset of the coronavirus pandemic forced higher education institutions across the world to pivot from face to face to remote teaching. For teaching methods that involve the transmission and dissemination of verbal/visual information between academic staff and students, video technologies provided immediate methods to respond to the restricted access to campus. Practical activities, that usually involve interaction with equipment, presented a greater challenge to adapt for remote delivery. With restrictions on higher education being partially lifted, many institutions worldwide intend to offer blended learning, prioritizing in-person activities that are troublesome to deliver online, such as practicals. Social distancing measures are reducing capacity and placing increased pressure on space, creating a need to optimise limited time students have in the lab and strategies to determine which activities can best utilize this limited resource. Time is constrained, leaving little opportunity to make radical changes to learning and teaching structures. In this publication, The department of Mulicdipalnary Engineering Education (MEE) at the University of Sheffield, utilise their experiences in practical teaching to provide simple, implementable ideas for blended practicals which maximize students' learning and experiences within the envelope of available resources.
\end{abstract}

\footnotetext{
${ }^{1}$ Corresponding Author: a.garrard@sheffield.ac.uk
} 


\section{Introduction}

At the start of 2020 the rapid onset of the coronavirus pandemic forced higher education institutions across the world to pivot from face to face to remote teaching. For teaching methods that involve the transmission and dissemination of verbal/visual information between academic staff and students, video technologies such as Zoom, Microsoft Teams and Blackboard Collaborate provided immediate methods to respond to the restricted access to campus. Practical activities, that usually involve interaction with equipment, presented a greater challenge to adapt for remote delivery.

The department of Mulicdipalnary Engineering Education (MEE) at the University of Sheffield is dedicated to the delivery of practical teaching for the Faculty of Engineering. Undistracted by other teaching and assessment methods, such as lectures, tutorials and exams, MEE focuses on practical teaching and has invested substantial effort innovating and optimizing the delivery of in-lab activities at scale [1]. Following the suspension of face to face teaching, 600 remaining practicals activities were translated from in-lab to remote format and selected examples were published [2]. A follow up publication categorizes tactics for delivering remote practicals, and presents their relative advantages and drawbacks to fulfil practical learning outcomes [3].

Prior to the suspension of face to face teaching in early 2020, all practical activities delivered by MEE were delivered as blended learning. With restrictions on higher education being partially lifted, many institutions worldwide intend to offer blended learning, prioritizing inperson activities that are troublesome to deliver online, such as practicals. With social distancing measures reducing capacity and placing increased pressure on space, there is currently a pressing need to optimise the limited time students have in the lab and to develop strategies to determine which activities can best utilize this limited resource.

Educators are now in the position of having to deliver practical teaching within a rapidly changing regulatory, financial and institutional landscape. Time is constrained, leaving little opportunity to make radical changes to learning and teaching structures. In this publication, MEE attempts to utilise its experiences of thinking deeply about practical teaching and provide simple, implementable ideas for blended practicals which maximize students' learning and experiences within the envelope of available resources.

\section{Teaching sandwich}

MEE consistently blends all practical teaching activities undertaken by engineering students by sandwiching the in-lab session between a pre-experimental activity and a post experimental activity. Completion of the pre-experimental activity is mandatory in order to gain access to the laboratory. As the process is uniformly applied to all activities, students quickly develop a habit of completing the tasks through repetition of the process. This in turn establishes a culture of students preparing for teaching sessions.

Pre-experimental activities typically involve preparation for the in-lab activity. Students can be made aware of the learning outcomes, the aims of the experiment, the procedure to follow, 
the operation of any equipment and any health and safety requirements. Students can also be asked to practice the techniques necessary to process and analyse the results. Engagement with the preparatory material, which is typically delivered online in text, video or interactive exercise formats, is assessed using a multiple choice test, which students must pass, called a "mandatory lab induction test", or MLIT.

Having students well prepared for practical teaching allows time spent interacting with equipment to be more fully utilised for the aims of the learning, rather than, for example, familiarisation with the equipment. In addition, there is a reduction in the number of low level questions during the in-lab session, such as operation of equipment or procedure. This not only reduces the amount of face to face interaction required, (in turn reducing costly staff time requirements and potential close contact resulting in spreading infection), but also frees up staff time to engage with deeper and more academically challenging questions and discussions with students. The MLITs can be adapted year on year, by identifying and including didactic instruction based on common questions from students from previous iterations of the teaching activity.

The rigorous pre-lab activity also eliminates the need to have a formal, verbal introduction to the laboratory activity. This is beneficial because laboratory spaces are often not well suited to presentation style teaching, and lecture-style introductions occupy valuable time when students could be interacting with the equipment. However, it has been found that a short "welcome" address, highlighting the key points with an overview of the activity provides a degree of "ceremony" to the activity, and grants "permission" for students, who otherwise may be unsure if they are allowed, to start experimental work.

The post experimental activity is typically a reflective piece of work to ensure the student considers the learning from their activity in context of their wider studies. The pre and post experimental activities can be specifically designed to act as a "bridge" between the classroom and laboratory based teaching, by including references to both [4].

\section{Threshold Assessment}

There are a number of reasons to summatively assess students, such as monitoring their progress or influencing their behaviour. Two distinct reasons within university courses are to ensure the fulfilment of the claimed learning outcomes, and to differentiate cohorts into categories of ability, e.g. first class, upper second...etc in the UK. Often summative assessment vehicles are designed to achieve both of these tasks simultaneously, for all module or programme learning outcomes. However, this assessment style is not a requirement. Establishing that learning outcomes have been met using a binary pass/fail assessment can be decoupled from a graded assessment that assesses all, some or, potentially, none of the learning outcomes. Reasons to implement threshold assessment include reducing assessment burden for staff and students, reducing student anxiety and simplifying a programme's core objectives. 
Some learning outcomes may define the quality of graduating students while others may imply meeting a competence. For example, engineering accrediting bodies may require experiences of workshop operations or understanding of health and safety practices [5]. With a threshold assessment approach, such learning outcomes could be identified as required by students to reach a minimum standard of competence to be considered safe to practice as an engineer, but expertise in itself wouldn't indicate mastery of the subject. Grading criteria which can be simplified to the binary states of met or not met are straightforward to write, communicate and assess against, allowing students and staff time to focus on more meaningful learning. Feedback on threshold assessments could still provide indications of performance, without the need to specify a grade which could influence the students' final degree classification. Threshold assessments also overcome variable expectations and interpretations by different markers. It is easier and more robust for teachers to simply decide whether or not someone has achieved a learning outcome rather than assigning precise numerical grades.

At the University of Sheffield, grades from individual modules are aggregated into a programme grade. A module mark of $40 \%$ is considered a pass and to achieve this all module learning outcomes need to be met. Further classifications are applied for attainment of certain grades above $40 \%$. An example of implementing threshold assessment on a module would be to provide one or more simple assessments collectively covering all module learning outcomes, marked on a pass/fail basis, which is resource efficient for academics and, with innovative assessment methods, could be automated. Successful completion of these threshold assessments would allow students to obtain a $40 \%$ pass mark for the module. If deemed appropriate, multiple attempts could be made until meeting the learning outcomes has been demonstrated. One or more further assessments, with a more sophisticated and granular grade criteria, can be provided and with the intention of differentiating the cohorts by demonstrating mastery of the subject.

When modules contain multiple assessments that all ensure both compliance with meeting learning outcomes and differentiation of standards among the cohort, the perception of continuous high stakes assessment can lead to student anxiety. With a threshold approach, students can be provided with an opportunity to ensure they have passed a module before undertaking a challenging differentiation assessment.

The extent to which learning outcomes should be assessed provides the opportunity for teaching teams to enter into discussion about the holistic aims of a programme. Contentious high-level analysis can determine the attributes that make a great engineer, which might identify learning outcomes such as: the ability to proficiently use mathematics, to abstract physical systems into analytical models or the employment of research/experimental methods as tools for which engineering need only demonstrate a degree of competence. Selecting only a few, key core defining qualities that demonstrate mastery of engineering, such as to apply creative thinking, innovation and implementation of solutions could be assessed with a few, capstone, programme level assessments. An additional advantage here is that if a learning outcome or topic is missed in an open-ended capstone task, it can easily be shown that a student has already met the required threshold elsewhere in their programme. Threshold assessment therefore aids thorough quality assurance. 


\section{Consider learning outcomes}

A teaching activity should be a vehicle for fulfilling specific learning outcomes. Educators invest significant effort in ensuring students have enjoyable experiences, challenging the strongest students while supporting the weakest. However, when reactive changes are required due to unforeseen circumstances, the most critical aspect to consider is maintaining the compliance of a programme by ensuring all learning outcomes are met. When redesigning a teaching activity to be practicable within a new context, the aim should be to consider the most effective method to meet the original learning outcomes, rather than the possible ways to replicate the activity, which are not necessarily the same.

MEE has a learning outcome framework for practical activities, which allows mapping of skill categories at a programme level and provides students with an opportunity to understand and reflect on how their learning dovetails into other taught materials. Every labsheet that MEE produces explicitly states on the cover page the learning outcomes for the activity, as shown in figure 1. Clearly identifying the required learning from any activity provided the starting point for designing a blended learning activity which would meet the same aims.

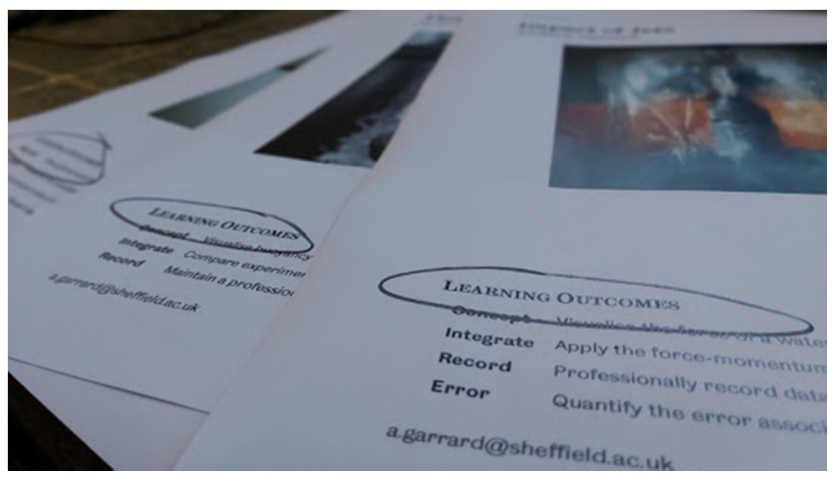

Figure 1: Learning outcomes for each activity explicitly stated on an every lab sheet.

Within the Fluids Engineering lab at the University of Sheffield, a purpose built "Osbourne Reynolds" experiential rig allows students to quantify the conditions required to generate "turbulent flow". However, students on the General Engineering programme were unable to perform this activity in-lab in spring 2020, due to the emergency suspension of all face to face teaching. The learning outcomes for this activity were to keep an experimental record of the activity, quantify the errors obtained in their measurements, and write a technical engineering report.

To fulfil these learning outcomes, students were asked to perform an equivalent experiment at home, using kitchen utensils. While the "at home" version didn't display the concepts with the same clarity as the bespoke rig, and the precision of the measured results was poor, there were no barriers to being able to record the procedure, observations and results, quantify the measurement errors, and write up the experimental findings. Some students also reported they found the experience "fun" given that all other practical activities were delivered online. The variation in the equipment available to students also generated some unanticipated creativity. To ensure that no students considered themselves disadvantaged, a candid video was provided 
by the activity leader to explicitly state that students were not being judged on their ability to produce precise results, but on their ability to quantify the error associated with the results that they obtained.

With reduced access to laboratory space, the session learning outcomes, rather than the activity itself, can be used to determine which activities to prioritise for in-lab delivery and which can be delivered remotely.

\section{Remote practicals playbook}

Reducing laboratory capacity means that only some learning outcomes will be able to be delivered with face to face teaching while others will have to be delivered remotely. After using the learning outcomes to decide which activities are best suited to face-to-face or remote activities, blended learning techniques can be selected to optimise student learning both inside and outside the laboratory. To help select tactics for delivering remote and blended practicals, MEE has developed the remote practicals playbook [6]. The objective of the playbook is to direct educators to particular categories of remote practical based on the learning outcomes that are required to be met. With an overview of the range of possible solutions to achieve learning outcomes remotely, a strategic selection of which learning outcomes are well suited to each delivery method can be made.

MEE plans to use the knowledge developed from designing remote practicals to deliver "remote enhanced practicals" as blended learning. By combining remote tactics as part of the pre and post experimental activities in the teaching sandwich, or alongside the session as a sister activity, the dual benefits of deeper learning and reduced contact time can be achieved.

\section{Balanced diet}

A wide range of tactics can be used to deliver blended practical learning, and ensuring balance of the different activities across a programme is critical. This is especially important when multiple modules requiring practical work are taken simultaneously. Although teaching activities, including practical exercises, are usually designed in self contained modules, students will experience a whole programme. To ensure their experience is engaging, enjoyable and manageable, a programme level overview must be taken to see the course through the eyes of a student. This will allow different types of blended activity to be mixed and matched across modules, maintaining a balance.

There are several teaching session characteristics to be balanced over time, including:

- Synchronous or asynchronous sessions;

- Remote practicals or face-to-face teaching on campus;

- Tactics of remote teaching (digital artefacts/simulations etc.);

- Cohort size and level of instructor interaction.

Communication between module leaders is essential to balance the programme e.g. do not set students simulation exercises for several weeks in a row, even on different module topics, 
without also using an in-person or synchronous online event to use a different skillset. Ensuring a balanced diet across modules can maintain momentum in the course, keep students engaged and inspired, and cater for a range of learning styles.

Figure 2 shows an example programme of practical activities for a single semester of an undergraduate engineering course. This includes practical activities from several different content modules. Notice that of the 9 practical activities, 3 of them are in-lab and they are spread evenly throughout the course. A blend of different teaching methods is used to deliver the activities, without using the same activity in succession. Importantly, the synchronous and asynchronous activities are also distributed through the semester, maintaining structure and real-time student contact.

\begin{tabular}{c|c|c|c|c|c|c|c|c|c}
\hline $\begin{array}{c}\text { Delivery } \\
\text { Method }\end{array}$ & In-lab & $\begin{array}{l}\text { Video } \\
\text { artefacts }\end{array}$ & $\begin{array}{l}\text { Remote } \\
\text { participation }\end{array}$ & $\begin{array}{l}\text { Video } \\
\text { artefacts }\end{array}$ & Simulation & In-lab & $\begin{array}{l}\text { Manufacture } \\
\text { by proxy }\end{array}$ & $\begin{array}{l}\text { Home kit } \\
\text { live session }\end{array}$ & In-lab \\
\hline $\begin{array}{c}\text { Synchronous or } \\
\text { asynchronous }\end{array}$ & S & A & S & A & A & S & A & S & S \\
\hline
\end{tabular}

$1 / 3$ in lab $\mathcal{C}$ Spread synchronous activities

Different tactics

Figure 2: An example programme of practical activities showing a good balance of activity delivery styles

After considering what can be delivered in a medium time period (a semester or academic year), consider the entire student programme over all three or four years to ensure that anything lost in remote practicals can be made up elsewhere. For instance, remote practicals may lose teamwork usually experienced when sharing lab equipment, or may lose the option for students to perform open-ended investigation above and beyond the expectations of a fixed lab exercise. By considering the entire programme, these learning requirements could be integrated with other activities e.g. teamwork lost in years 1 and 2 may mean more structured team building exercises are required in year 3 and 4 projects, but the skills are still learned at some point. This is particularly important if accreditation requirements must be satisfied.

\section{Simulations don't need to be costly}

An easy to implement method to translate an in-lab practical to a remote format is to provide numerical results obtained from the equipment for students to process as if they had obtained the data themselves. Amongst the drawbacks to this approach is the inability for students to employ a simple process of experimental design through setting a data capture strategy i.e. deciding which parameters are the independent, dependent and control variables and choosing value ranges for these parameters. This problem can be overcome by allowing students to simulate systems to obtain data for themselves.

Simulations are used to recreate the real-time cause and effect of a physical system in a digital environment. Often, when situations of experiments are discussed, systems using sophisticated 3D, VR or AR technologies are implied. These can be expensive to purchase or prohibitively time consuming to develop in-house. However, simple simulations that produce only numerical results can be developed with software designed for performing calculations, 
such as spreadsheets and programming languages. Systems can be set up to invite the user to specify input parameters and mathematical operations based on the underlying theory can produce the output. Logical operators can be used to test for specific conditions, for example results may only be displayed if buttons have been activated to represent turning on measurement equipment.

Simulations that only provide numerical output may lack realism, and may constrain the possible learning outcomes that can be taught. If results are produced instantly, numerous data points can be collected which fully resolve the relationship between the independent and dependent variable, whereas in the laboratory collecting results takes time and hence limits the amount of data that can be gathered. Delays can be placed into the production of results following the supply of input parameters, to replicate the process of data collection and force the need to carefully consider a data collection strategy. Further realism can be introduced using random functions to generate constrained variation in output data, to simulate experimental error and resulting in all students getting different results.

An example of this approach is shown in figure 3. A fluid mechanics experiment to measure the pressure drop in a number of piping components for various flow rates has been created using Google Sheets spreadsheet software. A schematic of the equipment is created by adjusting the border and shading of the cells, while background colours indicate where students should input parameters and where results are displayed. Crude animations are created by turning on and off background cell colours to represent the collection and flow of water. Using conditional statements, messages are displayed to students in the red box based on the actions they have performed, to provide instruction and information about the system status.

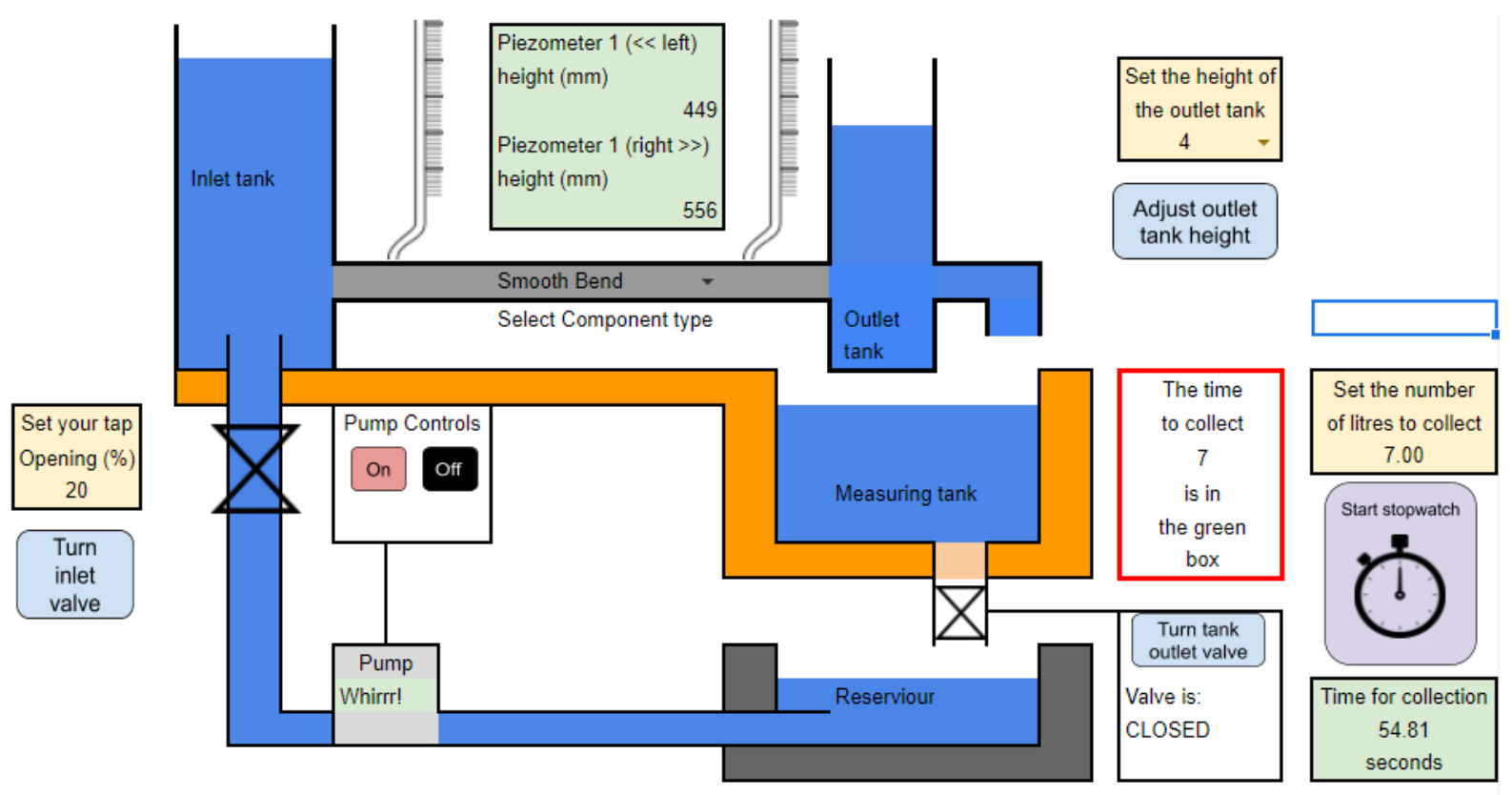

Figure 3: Example of simulated practical made using Google Sheets. 


\section{Synchronous remote participation}

If students are unable to be present in a laboratory to undertake an experiment, a sense of "event" can be created by a member of staff conducting the experiments while being filmed and streamed live to an audience. By creating a live experience, the session can contain interactive elements, such as question and answer, and can maintain the excitement of realtime cause and effect, even if the experiment is performed at a distance. This tactic has an additional advantage of being able to scale to large cohorts with little additional resource requirement.

Consideration should be given to recording and subsequently making the video footage available for later viewing. Retrospective viewing can be useful for revision, for students absent through extenuating circumstances, and for ensuring inclusivity for students with language barrier issues, who can slow down footage and review captions. However, providing asynchronous content could reduce the audience size and sense of community generated through a shared experience. A compromise could be the adaptive release of the recording to specific students with justified reasons for missing the original broadcast, although this would entail additional administrative burden.

If using synchronous remote participation, certain aspects are worth considering during the planning stages. Firstly, pre-experiment, preparatory material can be mandated as part of a teaching sandwich to ensure students gain maximum benefit from the session. This also reinforces the culture of preparing for attending teaching sessions, whether in-lab or remote.

Secondly, consider the resources that students will require during participation and explicitly communicate these sufficiently far in advance of the event to allow for them to be obtained. Active involvement during the broadcast, such as recording observations/data or processing results can replicate the experiences of conducting an experiment. However, in order to do this students may require a printed or digital copy of labsheets, the ability to annotate those sheets with digital software or with a pen, and other equipment such as a calculator or stopwatches. Teachers must ensure students are aware of these requirements and provide instructions for alternatives in case they can't access any particular resource.

Finally, if staff, space and equipment constraints allow, consider how multiple cameras and operators can create a more effective video for learning. When students engage with a piece of equipment in a laboratory, their attention can be placed on the entire system or focused into specific areas or aspects of the procedure. Different shots set up to focus on different aspects of the experiment will provide a greater replication of this experience, curated by the educator. Having a member of staff taking control of capturing the video will also allow the educator to interact with students, undistracted from the mechanics of operating the camera and, crucially for some activities, free up the use of their hands. An operator watching the live steam can also ensure framing, focus and lighting conditions are optimal and can make appropriate adjustments to enhance the quality of the video for the students. 


\section{Structuring asynchronous activities}

Asynchronous activities outside the laboratory have many advantages, such as the opportunity to engage with the work in a flexible way, making it inclusive for students with a range of external commitments and concentration issues, and eliminating the need to timetable the activity in a suitable room. However, students individually undertaking work without reactive support being available at all times requires careful activity design. While an in-lab activity is structured and paced by the cohort and teachers, during asynchronous practicals students will be required to take responsibility for structuring their own approach to working through an activity. Tactics can be employed to provide structure, which can assist students to plan and progress their work.

An activity should be broken down into small, discrete tasks, with specific milestones and with as much possibility for students to customise the experiences based on their ability as possible. Providing a logical layout of the task for students, in a chronological order, will immediately allow an identification of the number of steps, the order in which to complete them, and the size of the activity that they are undertaking.

Starting the activity with a "welcome" video can introduce the aim and purpose of the exercise, as well as describing the learning outcomes and how the teaching fits into their programme. Providing an enumerated list of tasks to complete and milestones to achieve at the start of the activity will help the students to structure their work.

Milestones can allow students to monitor their progress and identify their ability to fulfil the required tasks. They also provide an opportunity to allow students to customize their learning experience. If they are unable to complete tasks, they can be pointed to additional resources, such as further instructional materials or example answers. If these are still not sufficient, students can be pointed to where to obtain support.

To monitor cohort engagement and success, tasks can be automatically released only upon successful completion of particular milestones. For example, stock data could be supplied only after completing a quiz to establish understanding of the aims and procedure. Submission links for final assignments could be made available only after successful demonstration of data interpretation. Auditing the completion of task specific objectives will identify where cohorts require specific additional guidance or support.

\section{Maximising the investment of resource}

The transition to a blended learning strategy is advantageous for students and teachers but can also be costly both financially and in staff time. With proper planning, however, this needn't be the case. Many of the materials generated to support the transition away from exclusively face-to-face teaching, such as audio/video content and MILTs, can be adapted for multiple uses.

For example, video footage of equipment being operated, if shot in a clear cut manner with easily editable start and end sections, can be employed in a number of different areas. This 
could include, training for staff and graduate teaching assistants to use the equipment (including health \& safety), student lab induction (embedded within pre-experimental activities) and videos in lectures/tutorials related to the practicals, to provide a connection between the practical and classroom based teaching.

If curated carefully, most media content can be adapted to make a lab specific 'B-roll' (a collection of generic footage that can be edited in a modular fashion to create different videos). The advantage of this approach is that it can save a great deal of time in developing future teaching content, by minimising the need for re-recording. In some cases, if an update of the curriculum occurs, only a new vocal overdub or set of subtitles would be required, if there is no one speaking visibly to the camera. This strategy also has additional inclusivity benefits, as the language used can be translated for use by international students and the typeface/audio descriptions adapted for students with special educational needs.

The key to both of the above media reuse methods is using accurate and detailed metadata. In a rush to transition to online teaching, files can be stored with unhelpful filenames, in many different physical and cloud based storage locations. To combat this, setup a comprehensive folder structure by laboratory and then by piece equipment. In addition, embed as much data as possible within the media file, using tags and descriptions as well as titles. This will allow easy searching of the media assets at a later stage, when editing or producing new video content.

For institutions with little staff resource to generate this content, an alternative method could be to use 'stock' media or a subscription to an online platform such as The Journal of Visualized Experiments (JoVE) [7]. This approach has the benefit of immediate access to high quality content, but comes at the penalty of financial costs and a lack of educational specificity to directly link with module content.

Teaching material such as datasets can also be repurposed with the correct design. If a piece of equipment is thoroughly tested and calibrated by staff (or students), the data generated from this process can be used as a stock resource for interrogation and analysis for a number of educational activities at a later date, just as would be the case in the research field. A set of detailed step by step examples using this data, can be used in case studies, training for staff (especially useful for multidisciplinary work), comparison with simulation, formal exams and pre-experimental activities. Development of high quality blended learning opportunities can also be used to provide catch up opportunities for students missing original timetabled sessions, which can be administratively complex to reschedule.

The key to this process is to generate robust, high quality data in the first instance and a clear detailed explanation of the academic content with model answers, beyond this point any other modifications will only involve the removal of content, as the hard work is already done.

\section{Providing Staff Support}

As students become more experienced, lab activities are designed to be increasingly challenging, while, simultaneously, the level of support provided to them tends to decrease. 
When moving from a traditional face-to-face teaching setting towards a combination of remote and in-lab sessions, it is difficult to find the right balance between challenge for the student and support provided to them.

It is always beneficial to clarify what the students should see as a "challenge". The challenge should usually be defined as the pedagogical journey that students complete to meet the learning outcomes. This is a journey based on self-reflection and should not be unnecessarily cluttered with procedural complexity.

Teachers should provide sufficient support to students so that the transition from face-to-face to blended teaching will not become the "challenge". Despite the new teaching methods, or indeed any major change in programme delivery, students should remain focused on achieving the learning outcomes. However, this ideal focus on the defined challenge is often not achieved and plans should be made, ahead of term-time, for feedback strategies to assist struggling students. These forms of support will depend on several factors including student numbers, experience and diversity.

Emails arguably represent the most accessible tool students have to get in contact with academic staff. Students tend to like this method as it is "personal" and it does not expose students' doubts in front of their peers. Email requires no time before term time to set-up, but it becomes effective only when timely answers can be provided, making them a viable solution only with relatively small cohorts.

Online forums are also a common solution. In MEE, these are usually generated within the institutional Virtual Learning Environment (VLE) but external software can also be effectively used (e.g., Padlet). The forum can be made anonymous, as this may promote student engagement. In this scenario, it is also recommended that a suitable number of moderators are identified so that the forum is not misused. Moderators are usually selected among the teaching staff but, in an attempt to promote engagement, volunteers from the students can also be appointed. Forums can be managed so that other students can offer answers to open queries or by granting answering rights only to the teaching team, depending on students' capability to assist their peers. Forums tend to save time for the academic staff during term, so that instead of replying to multiple - and often similar - emails, they can instantaneously reach the whole cohort with a single answer to a query. Some students will find this solution less personal than emails, but others will thrive with the opportunity of getting in contact with all their peers. Forums should be set-up ahead of time and properly signposted to students.

Synchronous remote "drop-in" offers the possibility to engage in real-time with students. These sessions can be recorded (even though this may refrain some students from asking questions) and shared with the whole cohort. Alternatively, questions and answers can be compiled in a FAQ section of the VLE. It is good practice to ask in advance students for an expression of interest (not compulsory) to attend the session, as a high number of attendees may require splitting the remote session into groups each moderated by a member of the academic team. Additionally, it is effective to ask students ahead of time which questions they 
would like to be answered. This will allow the academic to better structure the answer and it will work as an ice-breaker for students that will then tend to come up with additional questions. 


\section{References}

1. Garrard, A and Beck, S.B.M. (2018) Pedagogical and cost advantages of a multidisciplinary approach to delivering practical teaching, The Interdisciplinary Future of Engineering Education Breaking Through Boundaries in Teaching and Learning, ed Kapranos. P.,Routledge

2. Bangert K, Bates J, Beck SBMB, Bishop ZK, Di Benedetti M, Fullwood J, et al. (2020) Remote Practicals in the Time of Coronavirus, a Multidisciplinary Approach 2020. doi:10.31224/osf.io/d7rkg.

3. Garrard, A., Bates, J., Beck, S.B.M and Funnell, A. C. (2020) Codifying an approach to remote practicals. doi: 10.20944/preprints202006.0182.v1

4. Garrard, A. \& Nichols, A. (2018). A Teaching Sandwich Approach to Integrating Classroom and Practical Teaching. 5th Annual Symposium of the United Kingdom \& Ireland Engineering Education Research Network: "Time for Change".

5. The Accreditation of Higher Education Programmes 3rd Edition, Engineeringcouncil.

https://www.engc.org.uk/engcdocuments/internet/Website/Accreditation $\% 20$ of $\% 20$ Higher\%20Education\%20Programmes\%20third\%20edition\%20(1).pdf (accessed 27/05/2020)

6. University of Sheffield Remote Practicals Playbook. https://sites.google.com/sheffield.ac.uk/remote-practicals-playbook/home (accessed $11 / 09 / 2020)$

7. Journal of Visualized Experiments https://www.jove.com/ (accessed 11/09/2020) 